\title{
Effects of cognitive load on neural and behavioral responses to smoking-cue distractors
}

\author{
R. Ross MacLean ${ }^{1,2,3}$ • Travis T. Nichols ${ }^{1}$ - James M. LeBreton ${ }^{1}$ - Stephen J. Wilson ${ }^{1,4}$
}

Published online: 24 March 2016

(C) Springer Science+Business Media New York (outside the US) 2016

\begin{abstract}
Smoking cessation failures are frequently thought to reflect poor top-down regulatory control over behavior. Previous studies have suggested that smoking cues occupy limited working memory resources, an effect that may contribute to difficulty achieving abstinence. Few studies have evaluated the effects of cognitive load on the ability to actively maintain information in the face of distracting smoking cues. For the present study, we adapted an fMRI probed recall task under low and high cognitive load with three distractor conditions: control, neutral images, or smoking-related images. Consistent with a limited-resource model of cue reactivity, we predicted that the performance of daily smokers $(n=17)$ would be most impaired when high load was paired with smoking distractors. The results demonstrated a main effect of load, with decreased accuracy under high, as compared to low, cognitive load. Surprisingly, an interaction revealed that the effect of load was weakest in the smoking cue distractor condition. Along with this behavioral effect, we observed significantly greater activation of the right inferior frontal gyrus (rIFG) in the low-load condition than in the high-load condition for trials containing smoking cue distractors. Furthermore, load-related changes in rIFG activation partially mediated the effects of load on task accuracy in the smoking-
\end{abstract}

Stephen J. Wilson

sjw42@psu.edu

1 Department of Psychology, Pennsylvania State University, 311 Moore Building, University Park, PA 16802, USA

2 VISN 1 Mental Illness Research, Education, and Clinical Centers, VA Connecticut Healthcare System, West Haven, CT, USA

3 Yale University School of Medicine, New Haven, CT, USA

4 Center for Brain, Behavior, and Cognition, Pennsylvania State University, University Park, PA, USA cue distractor condition. These findings are discussed in the context of prevailing cognitive and cue reactivity theories. These results suggest that high cognitive load does not necessarily make smokers more susceptible to interference from smoking-related stimuli, and that elevated load may even have a buffering effect in the presence of smoking cues under certain conditions.

Keywords Smoking · Working memory $\cdot$ Cognitive load · Interference $\cdot$ Cue reactivity

Most attempts to quit smoking cigarettes are unsuccessful (Piasecki, 2006). Quitting smokers may be prone to relapse in part because they lack the top-down cognitive control required to override salient, bottom-up impulses to smoke (Heatherton \& Wagner, 2011; Nestor et al., 2011). Among cognitive processes, working memory (i.e., the capacity to temporarily store and manipulate information) is particularly important for the top-down regulation of behavior because it allows one to resist the intrusion of unwanted or irrelevant information into active attention (Hofmann, Friese, Schmeichel, \& Baddeley, 2011; Ilkowska \& Engle, 2010). Recent findings have suggested that suboptimal working memory functioning hinders the ability to successfully quit smoking. Specifically, studies have demonstrated that the behavioral and neurobiological markers of deficient performance and/or inadequate effort during tasks thought to require working memory are associated with poor outcomes (e.g., more rapid relapse) in those trying to stop using cigarettes (see also Day et al. 2015; Hawkshead, Liebel, Schwarz, \& Sweet, 2014; Loughead et al., 2015; Patterson et al., 2010).

The use of working memory to support abstinence may be especially critical when quitting smokers encounter cigaretterelated stimuli (or smoking cues). Smoking cues reliably 
evoke a host of subjective and physiological responses in smokers (Carter \& Tiffany, 1999; Engelmann et al., 2012), including increases in the desire to smoke, an effect that is most pronounced following acute abstinence from cigarettes (Sayette, Martin, Wertz, Shiffman, \& Perrott, 2001; Sayette \& Tiffany, 2013). In addition to arousing a conscious desire to smoke, smoking cues can trigger implicit or automatic processes that promote cigarette use (e.g., Payne, McClernon, \& Dobbins, 2007; see also Tiffany \& Drobes, 1990; Wagner, Dal Cin, Sargent, Kelley, \& Heatherton, 2011; Waters et al., 2004). Presumably, the explicit and implicit responses prompted by smoking cues serve to make quitting smoking more difficult. In support of this view, naturalistic studies have indicated that smoking cues significantly increase the likelihood of relapse during smoking cessation attempts (Ferguson \& Shiffman, 2009; Shiffman, Paty, Gnys, Kassel, \& Hickcox, 1996). One way in which working memory can facilitate smoking cessation is by helping individuals prevent or counteract cue-elicited responses that would otherwise lead to smoking (e.g., by maintaining information related to the goal of achieving cigarette abstinence; Nichols \& Wilson, 2015).

It is well established that working memory is limited in capacity (see Cowan, 2005) and that the ability to use working memory to manipulate task-specific information while simultaneously ignoring irrelevant stimuli is highly related to the cognitive demands of the primary task. Presumably, this has significant implications for employing working memory to reduce the impact of salient smoking cues: The degree to which such efforts are successful is likely to depend on the availability of cognitive resources. According to load theory (Lavie, Hirst, de Fockert, \& Viding, 2004), higher working memory load impairs selective attention and increases distractor interference. Thus, when cognitive demands are low, interference is reduced due to more resources being available to concurrently maintain task performance and process distracting stimuli (for a review, see de Fockert, 2013). Several neuroimaging studies have highlighted the critical role of regions within the prefrontal cortex in the control of selective attention to task-specific stimuli (Corbetta \& Shulman, 2002; de Fockert, Rees, Frith, \& Lavie, 2004; de Fockert \& Theeuwes, 2012; Hopfinger, Woldorff, Fletcher, \& Mangun, 2001). For example, tasks that require selective attention to a target stimulus while ignoring irrelevant distractors are associated with greater frontal activation (as compared to tasks with no distractors; de Fockert et al., 2004). The increase in frontal activation is thought to reflect increased demands on top-down frontal control when an attention-capturing distractor is present. Therefore, the presence of competing stimuli can disrupt working memory by utilizing the same neural resources that are necessary for maintaining goaldirected behavior. Accordingly, the presence of a salient smoking cue can disrupt the top-down neural processes associated with the cognitive control necessary to sustain abstinence from smoking during a quit attempt.

The distracting potential of smoking cues is illustrated by research indicating that cue-elicited responses are resourcedemanding (i.e., responses to smoking-related stimuli "tie up" limited-capacity cognitive resources). For instance, secondary response time paradigms have shown that smokers take longer to respond to an auditory probe in the presence of smoking cues, relative to when they are in the presence of neutral cues (Cepeda-Benito \& Tiffany, 1996; Sayette \& Hufford, 1994). This dual-task effect is consistent with the idea that exposure to smoking cues decreases the availability of cognitive resources. Importantly, studies have also demonstrated that smoking cues disrupt the maintenance of information in memory (Heishman et al., 2006; Madden \& Zwaan, 2001; Wilson, Sayette, Fiez, \& Brough, 2007). On the basis of the premise that sufficient working memory resources are necessary to maintain task-relevant information, cue-elicited responses may be disruptive to smoking cessation in part because selective attention to cues ties up the very resources that are needed to support abstinence. Consistent with load theory, smoking cues are likely to be particularly problematic when individuals attempt to utilize limited-capacity working memory under taxing conditions (e.g., when trying to actively maintain a sizeable amount of information). In other words, one would expect that smoking cues would have the biggest impact on the performance of a particularly demanding working memory task. Insofar as effective performance requires considerable cognitive resources, leaving little or none to spare, any diversion of those resources by smoking cues may be very costly. In contrast, there may be sufficient resources to perform tasks that are not especially demanding, even when cognitive reserves are occupied by cue-elicited responses.

To date, these possibilities remain largely untested, because few studies have directly examined the effects of working memory load on the ability to actively maintain information in the face of distracting smoking cues. The goal of the present study was to address this important knowledge gap by characterizing the effect of cognitive load on behavioral and neural responses to smoking cues. By investigating performance using a probed recall task with visual distractors, we sought to evaluate task performance and identify the brain regions associated with cue processing and working memory at high and low cognitive loads using three distractor conditions: control (no distractor), neutral cues, and smoking cues. We expected that smoking cue distractors would interfere with limited working memory resources and that task performance would be most impaired on high-load trials paired with smoking-related stimuli. The reduction in selective attention to task-specific information in favor of salient smoking distractors under high cognitive load is consistent with load theory (Lavie et al., 2004). Additionally, we predicted that 
smoking distractors would be associated with increased activation in frontal regions associated with selective attention in the presence of distracting stimuli. Disentangling the relationships between cognitive processing and cue reactivity will help elucidate relationships that can inform clinical interventions and self-regulatory strategies.

\section{Method}

\section{Participants}

Daily cigarette smokers were recruited through advertisements in the community and local newspapers. We targeted a sample size of 16 participants, on the basis of the calculation that this would provide approximately $81 \%$ power to detect an effect of at least medium size $(f=0.25)$ given the study design, assuming a correlation of .5 among repeated measures and an alpha of .005 (a common threshold for statistical significance in functional magnetic resonance imaging [fMRI] research). Additional participants were recruited to allow for attrition and data loss. A total of 23 individuals were enrolled in the study. Of these, 17 yielded usable data (two participants were excluded for failing to meet $\mathrm{CO}$ requirements [which are detailed below], and four were excluded because of excessive head motion during the fMRI scanning). The participants were required to be right-handed native English speakers between the ages of 18 and 45 . In addition, they had to report that they had smoked at least 15 cigarettes per day for the past 24 months and to report that they were not currently planning to quit smoking or actively pursuing any form of smoking cessation treatment. Individuals were excluded if they (a) reported having a current psychiatric diagnosis, (b) reported having any chronic cardiac or respiratory issues, (c) indicated that they were currently taking any psychotropic medications that could influence brain responses, or (d) had any MRI contraindications. Participants' mean age was 26.5 years $(S D=$ 8.7 , range $=20-45$ years; $53 \%$ male, $47 \%$ female), and they reported smoking an average of 17.4 cigarettes per day $(S D=$ 3.8 , range $=15-30)$. Their mean Fagerstrom Test for Nicotine Dependence (FTND) score was $4.88(S D=1.6)$, suggesting a low to moderate level of dependence. The self-identified ethnicity of the sample was as follows: $94 \%$ Caucasian, $6 \%$ Hispanic. All procedures were approved by the institutional review board of the Pennsylvania State University, and written informed consent was obtained from all participants. Individuals were paid US\$50 for their participation.

\section{Materials}

Baseline assessment measures Demographic information and information regarding smoking patterns were assessed with standard forms (Wilson et al., 2012).
Smoking urge assessment Participants verbally rated their current urge to smoke on a scale ranging from 0 (absolutely no urge to smoke at all) to 100 (strongest urge to smoke I've ever experienced) twice during the experimental session: (1) immediately prior to being placed in the scanner for fMRI data acquisition, and (2) immediately before being removed from the scanner at the conclusion of fMRI data collection.

Task and stimuli Participants were scanned while performing a probed recall task adapted from prior research (Chein \& Fiez, 2010). E-Prime software (Psychological Software Tools, Pittsburgh, Pennsylvania) was used to control computerized stimulus presentation and the collection of responses and response latencies. Each trial of the task began with an encoding period during which a set of three (low-load trials) or six (high-load trials) English letters were presented sequentially at a rate of one item per second. The encoding period was followed by a delay period lasting $10 \mathrm{~s}$; participants were instructed to covertly maintain the list items throughout the delay. At the end of the delay, a memory probe consisting of a letter and number (e.g., $R-3$ ?) was presented for $3 \mathrm{~s}$. Participants pressed one of two buttons during the presentation of the memory probe to indicate whether or not the given letter was in the serial position indicated by the number in the list presented during the encoding period. Trials concluded with the presentation of a fixation cross for either $12 \mathrm{~s}$ (lowload trials) or $13 \mathrm{~s}$ (high-load trials), which allowed the hemodynamic responses evoked by the task to decay to baseline.

One of three different types of stimuli was presented during the delay of each trial: (1) an ellipsis (control [no distractor] condition); (2) a picture with smoking-related content (smoking cue condition); or (3) a picture with content similar to the smoking pictures, but devoid of smoking cues (neutral cue condition). The picture stimulus set consisted of 30 smoking-related and 30 neutral pictures, each presented once during the experiment. Pictures were selected from the International Smoking Image Series (ISIS; Gilbert \& Rabinovich, 1999), a standardized set of smoking images that have been used in several prior brain imaging studies (e.g., David et al., 2005; McClernon, Kozink, Lutz, \& Rose, 2009). Participants completed nine runs of the probed recall task, each containing ten randomly ordered trials (one or two trials per delay condition per memory load). Thus, the task consisted of a total of 90 trials (15 trials per delay condition per memory load).

\section{Procedure}

Individuals who responded to the recruitment advertisements completed a preliminary telephone screening interview. Eligible participants visited the laboratory for an initial baseline assessment session and an experimental session. During the baseline assessment session, participants provided an 
expired-air carbon monoxide (CO) sample, which was used to verify smoking status ( $>10$ parts per million; BreathCo, Vitalograph, Lenexa, Kansas). Participants also completed questionnaires collecting basic demographic information and details regarding their smoking behavior. After completing the baseline assessment, participants were scheduled for the experimental session. They were instructed to abstain from smoking and from using any nicotine-containing products for at least $12 \mathrm{~h}$ before the experiment, and that a $\mathrm{CO}$ sample would be obtained to verify compliance with these instructions. Participants also were instructed to refrain from consuming drugs or alcohol for the $24 \mathrm{~h}$ preceding the experiment.

Upon arrival for the experimental session, participants reported the last time they had smoked a cigarette, and a second CO sample was obtained to check compliance with the deprivation instructions. Participants had to have a $\mathrm{CO}$ level that was at least $50 \%$ lower than the initial sample provided during the baseline assessment session, a cutoff that was established on the basis of prior experience with similar samples and procedures (e.g., Wilson et al., 2012). The ranges of baseline and experimental $\mathrm{CO}$ values were $15-37$ and $2-15 \mathrm{ppm}$, respectively; the percentage reductions in CO ranged from $53 \%$ to $88 \%$. Recent research has suggested lower cutoffs to confirm $24 \mathrm{~h}$ of abstinence (Cropsey et al., 2014); however, the participants in the present study were only asked to refrain from smoking for $12 \mathrm{~h}$, and the observed postdeprivation $\mathrm{CO}$ values are generally consistent with recommended cutoffs for verifying overnight abstinence from smoking (see, e.g., Sandberg, Sköld, Grunewald, Eklund, \& Wheelock, 2011).

Following the $\mathrm{CO}$ verification, participants were instructed that they would not be able to smoke during the experiment and were given instructions about how to perform the probed recall task. They were informed that their main objective was to try to remember the serial order of the letters presented at the beginning of each trial. They also were told that they would be presented with pictures during the delay period of some trials (they were not informed that some of the pictures would have smoking-related content). To reduce the likelihood that they would try to avoid viewing the pictures (e.g., by turning away or shutting their eyes), the participants were directed to look at each picture because they would be asked some questions about them after completing the memory task. They were instructed that they did not have to do anything else regarding the pictures but look at them. Participants then completed a set of practice trials to ensure their understanding of the task demands. The practice set included both low-load and high-load trials, but with only ellipses and neutral picture presented during the delay period; no smoking pictures were presented during the practice trials. (Additional pictures without smoking content that were not part of the previously described set of 30 neutral pictures were used for the practice trials.)
After completing the practice set, participants rated their current urge to smoke and then were placed inside the scanner. Following collection of the anatomical images, participants completed the probed recall task while fMRI data were collected. A second urge rating was collected from participants immediately after they had completed the final run of the task. Finally, the participants were removed from the scanner and debriefed.

fMRI data acquisition Scanning was conducted at the Penn State Social, Life, and Engineering Sciences Imaging Center using a research-dedicated 3-T Siemens Magnetom Trio scanner (Siemens Medical Solutions, Erlangen, Germany). Prior to functional scanning, a high-resolution $(1 \times 1 \times 1 \mathrm{~mm}$ voxels $)$ three-dimensional structural volume was collected using a magnetization-prepared rapid gradient-echo sequence. Next, 38 -slice oblique-axial functional images $(3 \times 3 \times$ $3 \mathrm{~mm}$ voxels) were acquired using a standard echo-planar imaging pulse sequence $(\mathrm{TR}=2,000 \mathrm{~ms}, \mathrm{TE}=25 \mathrm{~ms}$, FOV $=192 \mathrm{~mm}$, flip angle $=79^{\circ}$ ). Participants completed a total of nine 5-min functional runs, with 150 functional images being collected during each run.

fMRI data analysis BrainVoyager QX software (version 2.4.2; Brain Innovation, Maastricht, The Netherlands) was used to preprocess and analyze the imaging data. The following preprocessing steps were employed prior to the statistical analysis: motion correction (six-parameter rigid-body transformation), slice scan time correction (trilinear/sync interpolation), spatial smoothing using a three-dimensional Gaussian filter (4-mm full width at half maximum), voxel-wise linear detrending, and high-pass filtering of frequencies (three cycles per time course). Subsequently, structural and functional images were transformed to standard Talairach stereotaxic space (Talairach \& Tournoux, 1988).

We used a two-level approach adapted from prior research to analyze the fMRI data collected during the probed recall task (e.g., Chein \& Fiez, 2010; Yoon, Curtis, \& D’Esposito, 2006; Zarahn, Aguirre, \& D’Esposito, 1999). First, a voxelwise (i.e., whole-brain) general linear model (GLM) was used to estimate the fMRI signal changes associated with the temporally distinct phases of the memory task and the various trial conditions at the individual-participant level. The GLM contained a total of nine task-related regressors, each modeled using a boxcar function convolved with a standard twogamma hemodynamic response function: regressors for the encoding phases of low-load (3-s duration) and high-load (6$\mathrm{s}$ duration) trials; six regressors for the delay phases of trials (control, neutral cue, and smoking cue conditions, each under low and high load; 10-s duration); and a regressor for the memory probe/response phase of trials (3-s duration). The GLM also included the six motion parameters obtained during motion correction as covariates of no interest. 
Second, select parameter estimates obtained at the individual-participant level were entered into a randomeffects group analysis. Our primary goal was to compare the effects of neutral cue and smoking cue distractors on brain activity during working memory maintenance, and more specifically, to evaluate the extent to which these effects were modulated by memory load. Accordingly, the group-level analysis focused on the covariates modeling the delay periods of the probed recall task (covariates for the other task phases were included in the model at the individual-participant level to account for the variance associated with processing occurring before and after the delay period; see Chein \& Fiez, 2010; Zarahn et al., 1999). Specifically, using parameter estimates for the delay period of the probed recall task as the dependent measures of interest, we conducted a voxel-wise repeated measures analysis of variance (ANOVA) with Memory Load (high, low) and Delay Condition (control, neutral cue, smoking cue) as within-participants factors and Participant as a random factor.

The BrainVoyager QX Cluster-Level Statistical Threshold Estimator plugin (which implements a Monte Carlo simulation approach to multiple-comparisons correction) was used to determine the appropriate threshold for the group statistical maps (Goebel, Esposito, \& Formisano, 2006). After 1,000 iterations, it was determined that a corrected mapwise falsepositive rate of $p<.05$ would be obtained by combining a pervoxel threshold of $p<.005$ with cluster-extent thresholds of 16,12 , and 11 contiguous voxels for the statistical maps corresponding to the main effect of memory load, the main effect of delay condition, and the Memory Load $\times$ Delay Condition interaction, respectively. Only clusters meeting these criteria were considered significant.

\section{Results}

\section{Smoking urge}

As we described above, participants reported their urge to smoke using a 0-100 scale prior to being placed in the scanner and just after completion of the probed-recall task. Although the mean urge levels rose over the course of the study as expected, the results indicated that the pretask $(M=62.7, S D$ $=26.9)$ and posttask $(M=70.5, S D=28.4)$ urge ratings did not differ significantly, $t(16)=1.53, p=.15, d=0.77$.

\section{Behavioral performance}

We conducted a 2 (Memory Load: low-load, high-load) $\times 3$ (Delay Condition: control, neutral cue, smoking cue) repeated measures ANOVA with average accuracy as the dependent measure. Both the main effect of memory load, $F(1,16)=$ 23.76, $p<.001, \eta_{\mathrm{G}}{ }^{2}=.27$, and the main effect of delay condition, $F(2,32)=3.88, p=.03, \eta_{\mathrm{G}}{ }^{2}=.02$, were significant. These effects were qualified, however, by a significant Memory Load $\times$ Delay Condition interaction, $F(2,32)=$ $3.53, p=.04, \eta_{\mathrm{G}}{ }^{2}=.04$. To characterize the nature of this interaction, we tested the simple main effect of load separately for each delay condition, with each yielding a significant result: control trials, $F(1,16)=15.82, p=.001, \eta_{\mathrm{G}}{ }^{2}=.29$; neutral cue trials, $F(1,16)=20.55, p<.001, \eta_{\mathrm{G}}{ }^{2}=.35$; smoking cue trials, $F(1,16)=6.53, p=.02, \eta_{\mathrm{G}}^{2}=.15$. Specifically, participants were less accurate during high relative to low memory load across delay conditions, but the increase in memory load had the weakest effect on accuracy for trials containing smoking cues (see Table 1).

To explore whether this effect was related to the clinically meaningful smoking variables, we examined the association between load-related changes in performance (accuracy during high-load smoking cue trials minus accuracy during lowload smoking cue trials) and both self-reported craving and level of nicotine dependence. Load-related changes in accuracy for the trials containing smoking cues were not related to either the pretask $[r(15)=-.01, p=.98]$ or posttask $[r(15)=$ $.06, p=.81]$ urge ratings. Similarly, changes in accuracy for smoking cue trials were not related to FTND scores, $r(15)=$ $-.18, p=.47$.

\section{fMRI results}

Main effect of memory load The brain regions exhibiting a main effect of memory load are summarized in Table 2 and depicted in Fig. 1. Delay-related activation was greater under high than under low memory load in several areas, including the dorsal anterior cingulate (ACC), dorsal and lateral portions of the prefrontal cortex, precentral gyrus, inferior parietal lobe, and anterior insula. Greater delay-related activation during low than during high memory load was observed in the subgenual ACC, medial portions of the prefrontal cortex, inferior frontal gyrus, posterior cingulate gyrus, temporal gyrus, occipital gyrus, fusiform gyrus, and posterior insula.

Main effect of delay condition A significant main effect of delay condition was observed in several brain areas, including a large extent of occipital visual cortex, multiple sites in the

Table 1 Means (and standard deviations) for percent accuracy as a function of memory load and delay condition

\begin{tabular}{lll}
\hline & \multicolumn{2}{l}{ Memory Load } \\
\cline { 2 - 3 } Delay Condition & Low Load & High Load \\
\hline Control & $96.1(8.8)$ & $76.9(20.0)$ \\
Neutral cue & $94.6(6.3)$ & $74.6(18.8)$ \\
Smoking cue & $93.5(6.9)$ & $84.8(13.4)$ \\
\hline
\end{tabular}


Table 2 Brain regions exhibiting a main effect of memory load

\begin{tabular}{|c|c|c|c|c|c|c|c|}
\hline \multirow[b]{2}{*}{ Region } & \multirow[b]{2}{*}{ Size $\left(\mathrm{mm}^{3}\right)$} & \multicolumn{3}{|c|}{ Talairach Coordinates } & \multirow[t]{2}{*}{ Average $F$ Ratio } & \multicolumn{2}{|c|}{$M(S D) \%$ Signal Change } \\
\hline & & $x$ & $y$ & $z$ & & Low Load & High Load \\
\hline \multicolumn{8}{|l|}{ High Load > Low Load } \\
\hline Anterior cingulate (dorsal)/medial frontal gyrus & 7,015 & -10 & 16 & 42 & 16.72 & $0.10(0.11)$ & $0.21(0.12)$ \\
\hline Right insula (anterior) & 2,386 & 32 & 16 & 3 & 16.41 & $0.06(0.09)$ & $0.17(0.12)$ \\
\hline Left insula (anterior) & 1,449 & -31 & 25 & 6 & 13.73 & $0.10(0.09)$ & $0.19(0.13)$ \\
\hline Right middle frontal gyrus/superior frontal gyrus & 5,352 & 32 & 28 & 39 & 13.29 & $0.01(0.11)$ & $0.14(0.17)$ \\
\hline Left precentral gyrus & 1,458 & -50 & -8 & 54 & 13.23 & $0.21(0.23)$ & $0.39(0.30)$ \\
\hline Right inferior parietal lobule & 519 & 39 & -50 & 39 & 13.17 & $0.03(0.14)$ & $0.13(0.16)$ \\
\hline Right middle frontal gyrus/medial frontal gyrus & 886 & 23 & 1 & 47 & 12.64 & $0.01(0.06)$ & $0.07(0.08)$ \\
\hline Left inferior parietal lobule & 608 & -34 & -53 & 39 & 12.59 & $0.10(0.16)$ & $0.22(0.21)$ \\
\hline Left middle frontal gyrus & 2,522 & -46 & 22 & 31 & 12.55 & $0.11(0.11)$ & $0.22(0.14)$ \\
\hline \multicolumn{8}{|l|}{ Low Load > High Load } \\
\hline Right middle temporal gyrus/superior temporal gyrus & 1,740 & 53 & -68 & 24 & 15.06 & $-0.04(0.17)$ & $-0.17(0.20)$ \\
\hline Left middle temporal gyrus/middle occipital gyrus & 4,453 & -53 & -77 & 27 & 14.11 & $-0.04(0.13)$ & $-0.20(0.21)$ \\
\hline Left insula (posterior)/superior temporal gyrus & 1,387 & -36 & -14 & 0 & 12.79 & $-0.05(0.09)$ & $-0.11(0.11)$ \\
\hline Medial frontal gyrus/superior frontal gyrus & 4,233 & -4 & 55 & 24 & 12.78 & $0.04(0.12)$ & $-0.11(0.21)$ \\
\hline Right inferior occipital gyrus/fusiform gyrus & 1,051 & 32 & -92 & -15 & 12.56 & $0.41(0.30)$ & $0.20(0.28)$ \\
\hline Right middle temporal gyrus/superior temporal gyrus & 673 & 62 & -44 & 9 & 12.51 & $-0.02(0.15)$ & $-0.14(0.20)$ \\
\hline Posterior cingulate gyrus & 721 & -1 & -20 & 45 & 12.09 & $-0.03(0.14)$ & $-0.12(0.16)$ \\
\hline Right insula (posterior)/superior temporal gyrus & 782 & 47 & -5 & -3 & 12.08 & $0.00(0.10)$ & $-0.06(0.10)$ \\
\hline Anterior cingulate (subgenual)/medial frontal gyrus & 1,530 & -1 & 16 & -6 & 11.95 & $0.10(0.11)$ & $-0.08(0.17)$ \\
\hline Left superior temporal gyrus & 438 & -55 & 16 & -21 & 11.85 & $0.05(0.09)$ & $-0.03(0.09)$ \\
\hline
\end{tabular}

Stereotaxic coordinates are given for local maxima of activation clusters in Talairach atlas space. $\%$ signal change $=$ percent change in the BOLD signal relative to the estimated baseline

prefrontal cortex, dorsal and ventral ACC, and temporal and parietal regions (see Table 3 and Fig. 2). Follow-up pairwise comparisons (Benjamini-Hochberg-corrected with the false discovery rate set at .05; Benjamini \& Hochberg, 1995) revealed a consistent pattern, such that delay-related activation during control trials differed from the delay-related activation during both trials containing neutral cues and trials containing cigarette cues for each of these regions (see Table 4). For most of these regions, activation during the delay period was greater for trials containing distractors (neutral or smoking cues) than for control trials. With one exception (a region encompassing portions of the inferior parietal lobe, angular gyrus, and

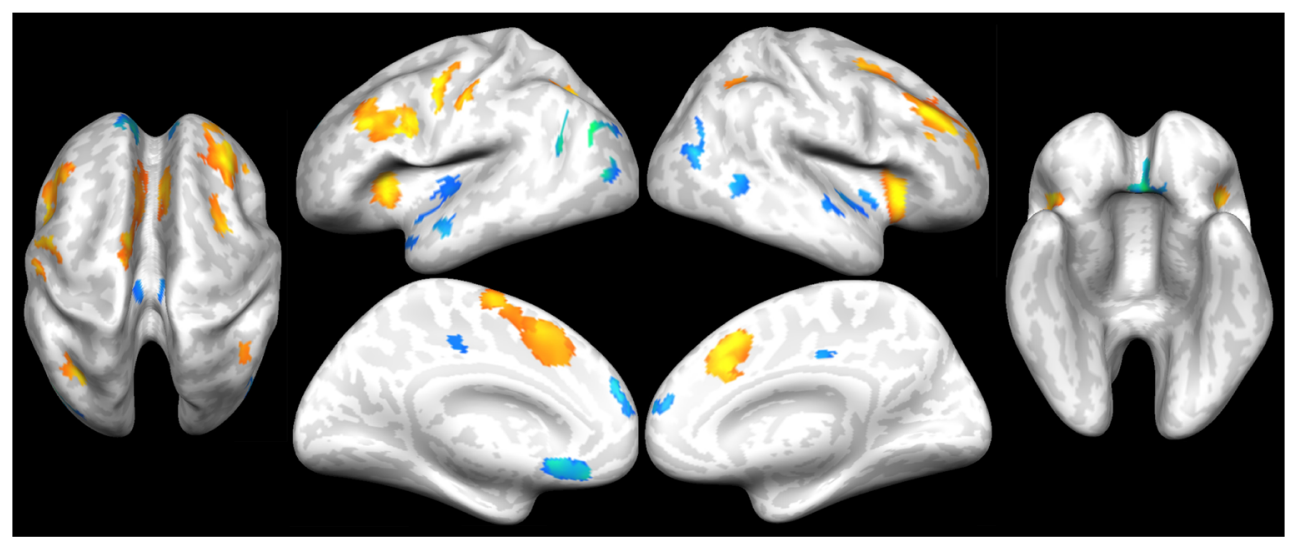

Fig. 1 Regions exhibiting a significant main effect of memory load. The activation map is shown on an inflated cortical surface. Areas for which delay-related activation was greater under high than under low memory load are depicted in "warm" colors (yellow/orange); those for which delay-related activation was greater under low than under high memory load are depicted in "cool" colors (blue/green). (See online for the color version) 
Table 3 Brain regions exhibiting a main effect of delay condition

\begin{tabular}{|c|c|c|c|c|c|c|c|c|}
\hline \multirow[b]{2}{*}{ Region } & \multirow[b]{2}{*}{ Size $\left(\mathrm{mm}^{3}\right)$} & \multicolumn{3}{|c|}{ Talairach Coordinates } & \multirow[t]{2}{*}{ Average $F$ Ratio } & \multicolumn{3}{|c|}{$M(S D) \%$ Signal Change } \\
\hline & & $x$ & $y$ & $z$ & & Ctrl & $\mathrm{Neu}$ & Smk \\
\hline $\begin{array}{l}\text { Bilateral cuneus/middle occipital gyrus/lingual } \\
\text { gyrus/fusiform gyrus/parahippocampal gyrus }\end{array}$ & 238,827 & 20 & -71 & -12 & 27.87 & $0.03(0.10)$ & $0.58(0.20)$ & $0.59(0.21)$ \\
\hline $\begin{array}{l}\text { Medial frontal gyrus/superior frontal gyrus/anterior } \\
\text { cingulate (pregenual/subgenual) }\end{array}$ & 33,361 & -1 & 55 & -13 & 11.14 & $-0.11(0.11)$ & $0.04(0.15)$ & $0.07(0.12)$ \\
\hline Left inferior frontal gyrus/superior temporal gyrus & 9,589 & -37 & 28 & -11 & 9.58 & $-0.07(0.15)$ & $0.11(0.19)$ & $0.12(0.12)$ \\
\hline Left middle frontal gyrus/inferior frontal gyrus & 8,564 & -55 & 19 & 30 & 9.32 & $0.10(0.13)$ & $0.26(0.18)$ & $0.21(0.15)$ \\
\hline $\begin{array}{l}\text { Right middle frontal gyrus/inferior frontal gyrus/ } \\
\text { precentral gyrus }\end{array}$ & 17,878 & 50 & 34 & 9 & 8.53 & $0.01(0.12)$ & $0.17(0.17)$ & $0.16(0.16)$ \\
\hline $\begin{array}{l}\text { Right inferior parietal lobule/angular gyrus/superior } \\
\text { temporal gyrus }\end{array}$ & 7,651 & 47 & -62 & 45 & 8.34 & $0.00(0.17)$ & $-0.17(0.23)$ & $-0.10(0.19)$ \\
\hline Right precuneus/superior parietal lobule & 746 & 29 & -53 & 48 & 8.1 & $0.06(0.12)$ & $0.18(0.18)$ & $0.16(0.15)$ \\
\hline Left parahippocampal gyrus/amygdala & 616 & -19 & -5 & -12 & 8.08 & $-0.04(0.13)$ & $0.09(0.15)$ & $0.09(0.16)$ \\
\hline Left middle temporal gyrus/inferior temporal gyrus & 1,194 & -67 & -11 & -12 & 7.97 & $-0.09(0.11)$ & $0.00(0.13)$ & $0.03(0.11)$ \\
\hline Right middle frontal gyrus/superior frontal gyrus & 1,349 & 32 & 25 & 42 & 7.75 & $0.07(0.10)$ & $-0.01(0.14)$ & $-0.02(0.10)$ \\
\hline Right middle temporal gyrus/inferior temporal gyrus & 817 & 62 & -23 & -12 & 7.34 & $0.04(0.15)$ & $-0.07(0.15)$ & $-0.03(0.10)$ \\
\hline
\end{tabular}

Stereotaxic coordinates are given for local maxima of activation clusters in Talairach atlas space. $\%$ signal change $=$ percent change in the BOLD signal relative to the estimated baseline, $\mathrm{Ctrl}=$ control trials [no distractor], $\mathrm{Neu}=$ neutral cue, $\mathrm{Smk}=$ cigarette cue

superior temporal gyrus in the right hemisphere), we observed no difference in delay period activation between trials containing neutral cues and trials containing cigarette cues.

Memory Load $\times$ Delay Condition interaction Our central aim was to identify regions for which the interaction between working memory load and delay condition was significant. As is depicted in Fig. 3, such an effect was observed in the right inferior frontal gyrus (rIFG; Talairach coordinates: $x=47, y=$ $37, z=15$; size $=415 \mathrm{~mm}^{3}$; average $F$ ratio $\left.=8.06\right)$. We tested the simple main effect of memory load separately for each delay condition to decompose the interaction. There was no difference in delay-related activation under low-load versus high-load trials in the control trials, $F(1,16)=0.20, p=.66$, $\eta_{\mathrm{G}}{ }^{2}=.00$, or the trials containing neutral cues, $F(1,16)=0.17$, $p=.68, \eta_{\mathrm{G}}{ }^{2}=.00$. In contrast, we did observe a significant effect of load on delay-related activation for trials containing smoking cues, $F(1,16)=22.81, p<.001, \eta_{\mathrm{G}}{ }^{2}=.40$.

As with the behavioral performance, we also assessed the relationship between the effects of load on activation of the rIFG and the smoking-related variables. Load-related changes in rIFG activation for trials containing smoking cues were not related to pretask $[r(15)=-.02, p=.94]$ or posttask $[r(15)=$ $-.18, p=.49$ ] urge ratings. Likewise, we found no association between changes in rIFG activation during smoking cue trials and FTND scores, $r(15)=.08, p=.75$.

\section{Exploratory mediation analysis}

As we described above, memory load had an effect on both behavioral accuracy and delay-related activation of the rIFG for trials containing smoking cues. Furthermore, the effect of memory load was in the same direction in each case (i.e.,

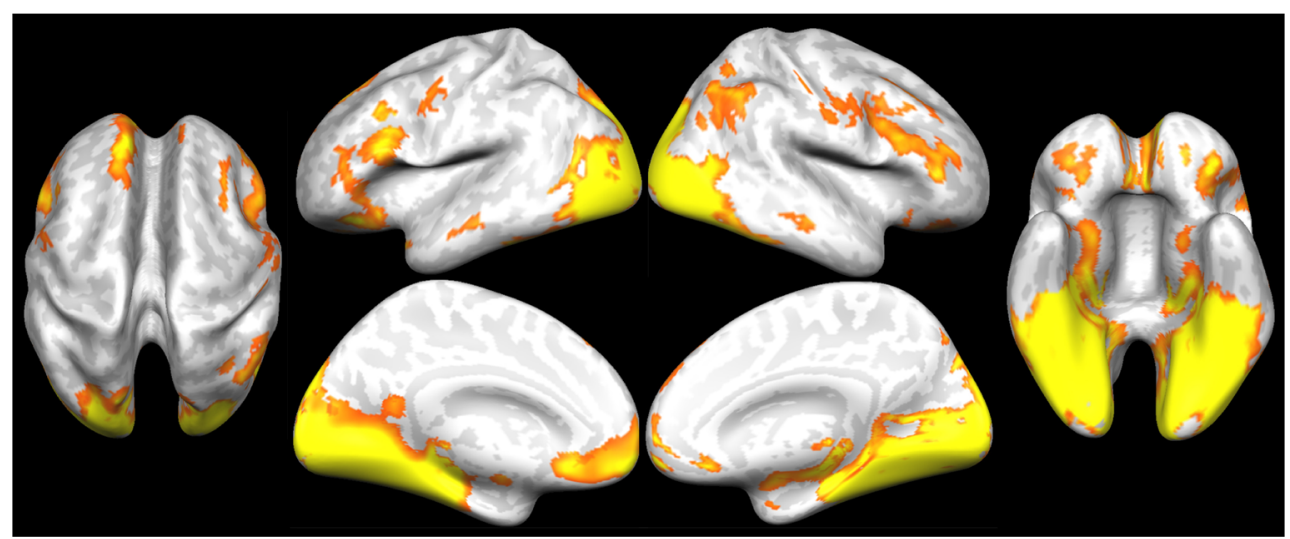

Fig. 2 Regions exhibiting a significant main effect of delay condition. The activation map is shown on an inflated cortical surface. (See online for the color version) 
Table 4 Results of pairwise comparisons for regions exhibiting a main effect of delay condition

\begin{tabular}{|c|c|c|c|c|c|c|c|c|c|}
\hline & \multicolumn{3}{|c|}{ Ctrl vs. Neu } & \multicolumn{3}{|c|}{ Ctrl vs. Smk } & \multicolumn{3}{|c|}{ Neu vs. Smk } \\
\hline & $F$ & $p$ & $p^{*}$ & $F$ & $p$ & $p^{*}$ & $F$ & $p$ & $p^{*}$ \\
\hline Bilateral cuneus/middle occipital gyrus/lingual gyrus/fusiform gyrus/parahippocampal gyrus & 124.03 & $<.001$ & $<.001$ & 88.37 & $<.001$ & $<.001$ & 0.02 & .88 & .91 \\
\hline Medial frontal gyrus/superior frontal gyrus/anterior cingulate (pregenual/subgenual) & 34.79 & $<.001$ & $<.001$ & 40.36 & $<.001$ & $<.001$ & 1.06 & .32 & .39 \\
\hline Left inferior frontal gyrus/superior temporal gyrus & 38.3 & $<.001$ & $<.001$ & 30.34 & $<.001$ & $<.001$ & 0.11 & .74 & .79 \\
\hline Left middle frontal gyrus/inferior frontal gyrus & 35.56 & $<.001$ & $<.001$ & 18.85 & $<.001$ & $<.01$ & 2.89 & .11 & .15 \\
\hline Right middle frontal gyrus/inferior frontal gyrus/precentral gyrus & 39.33 & $<.001$ & $<.001$ & 21.22 & $<.001$ & $<.001$ & 0.48 & .5 & .57 \\
\hline Right inferior parietal lobule/angular gyrus/superior temporal gyrus & 19.5 & $<.001$ & $<.001$ & 15.09 & $<.01$ & $<.01$ & 7.29 & .02 & .02 \\
\hline Right precuneus/superior parietal lobule & 12.83 & $<.01$ & $<.01$ & 12.18 & $<.001$ & $<.001$ & 0.72 & .41 & .48 \\
\hline Left parahippocampal gyrus/amygdala & 19.89 & $<.001$ & $<.001$ & 10.04 & $<.01$ & $<.01$ & $<0.01$ & .97 & .97 \\
\hline Left middle temporal gyrus/inferior temporal gyrus & 11.68 & $<.01$ & $<.01$ & 17.25 & $<.001$ & $<.01$ & 1.44 & .25 & .31 \\
\hline Right middle frontal gyrus/superior frontal gyrus & 10.62 & $<.01$ & $<.01$ & 25.48 & $<.001$ & $<.001$ & 0.21 & .65 & .72 \\
\hline Right middle temporal gyrus/inferior temporal gyrus & 28.25 & $<.001$ & $<.001$ & 8.04 & .01 & .02 & 2.81 & .11 & .15 \\
\hline
\end{tabular}

$\mathrm{Ctrl}=$ control trials $\left[\right.$ no distractor], $\mathrm{Neu}=$ neutral cue, $\mathrm{Smk}=$ cigarette cue, $p=$ uncorrected $p$ value, $p^{*}=$ Benjamini-Hochberg-corrected $p$ value

greater accuracy and delay-related rIFG activation, respectively, during low relative to high memory load). This pattern of effects satisfies the criteria required for testing mediation in a within-participants experimental design (Judd, Kenny, \& McClelland, 2001). Accordingly, using the procedure outlined by Judd and colleagues, we conducted exploratory analyses examining whether the effect of memory load on accuracy was mediated by load-related changes in activation of the rIFG. To explore the viability of a within-person mediation model, we first computed three new variables: (1) a difference score variable was computed on the outcome variable (behavioral accuracy) by subtracting each participant's score in the low-memory-load condition from his or her score in the highmemory-load condition; (2) a similar difference score variable was computed for the potential mediator (activation of the rIFG); and finally, (3) a unit-weighted composite sum score variable was computed by adding together the scores on the potential mediator (activation of the rIFG) obtained in the low- and high-memory-load conditions. The latter variable was also centered about the sample mean.

Following the suggestions of Judd et al. (2001), we regressed the behavioral accuracy difference score onto the activation difference score and the (mean-centered) activation sum score. The mean difference in rIFG activation was a significant predictor of the mean difference in accuracy $[B=-$ $0.31 ; t(14)=-2.24, p=.04, d=1.20]$, indicating a significant mediation effect. The intercept of the model was also significant $[B=-0.17 ; t(14)=-3.51, p<.01, d=1.88]$, indicating that the treatment (memory load) exerted an effect on accuracy over and above load-related differences in rIFG activation. Finally, the mean-centered sum of rIFG activation was not a significant predictor of the mean difference in accuracy $[B=$ $0.16 ; t(14)=0.13, p=.90, d=0.07]$, suggesting that activation of the rIFG did not moderate the effect of memory load on accuracy. Altogether, this pattern of results is consistent with a model in which load-related changes in activation of the rIFG

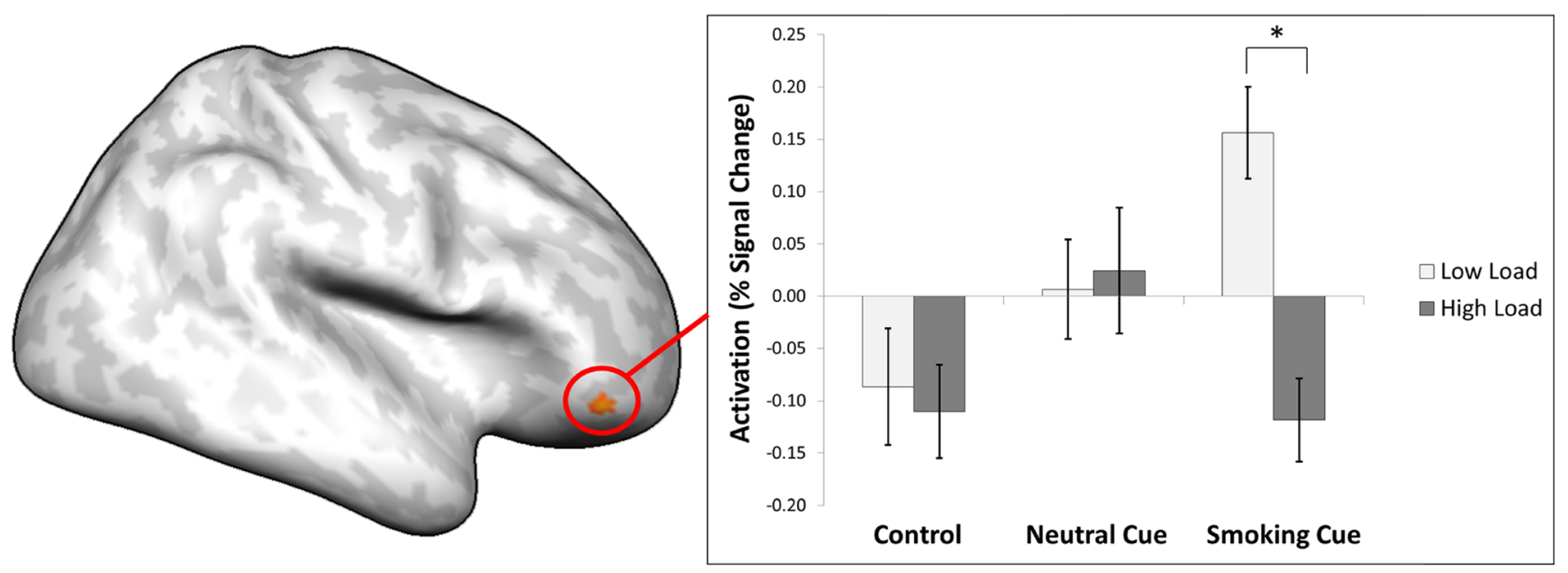

Fig. 3 Left: Region of the right inferior frontal gyrus (rIFG) exhibiting a significant Memory Load $\times$ Delay Condition interaction. The activation map is shown on an inflated cortical surface. Right: Delay-related activation of the rIFG as a function of memory load (high, low) and delay condition (control, neutral cue, cigarette cue). The asterisk denotes a significant difference between conditions 
partially mediate the effects of memory load on task performance during smoking cue trials.

\section{Discussion}

The main objective of the study was to investigate the effects of cognitive load on behavioral and neural responses to cigarette-related distractors. Related to this aim, we adapted an fMRI probed recall task with two levels of cognitive load each with three delay conditions: control (no distractor), neutral images, or smoking-related images. As expected, we observed a robust main effect of memory load, such that participants performed more poorly in the high-load than in the lowload condition, irrespective of delay condition. We also observed a significant interaction between memory load and delay condition. Specifically, whereas participants were less accurate during high than during low memory load across delay conditions, this effect was weakest for trials containing smoking cues during the delay period. Smoking cues thus appeared to mitigate the extent to which performance was degraded by increasing memory load. This finding is contrary to our expectation, based on load theory, that distracting smoking cues would be most detrimental to performance in the high-load condition. Instead, the present results suggest that smoking-related imagery may not automatically occupy limited working memory resources and impair cognitive processing during the performance of an effortful task.

The surprising finding that smoking-related cues dampened the influence of memory load on accuracy stands in contrast to previous research that highlights the detrimental impact of drug-related cues on task performance in addicted populations (Cox, Fadardi, \& Pothos, 2006; Ehrman et al., 2002; Evans, Craig, Oliver, \& Drobes, 2011; Hester \& Garavan, 2009; Madden \& Zwaan, 2001). However, whereas addiction research has traditionally focused on the ways in which drug cues can interfere with cognitive processing, emerging theory and research have emphasized the notion that the opposite can also occur: Ongoing cognitive processing can attenuate responses to drug cues. The idea that cognitive processing can blunt cue reactivity is a direct extension of the central tenets of the elaborated intrusion (EI) theory developed by Kavanagh, Andrade, and May (2005). The EI theory posits that the state of desire is initiated by automatic thoughts (e.g., craving prompted by exposure to smoking-related cues), which is followed by cognitive elaboration - the mental embellishment of the initial automatic thoughts by seeking relevant information in the environment. Importantly, this elaborated information is retained in working memory and manipulated through the use of nonautomatic (effortful or controlled) cognitive processes, such as visual imagery (Kavanagh et al., 2005). For example, seeing an ashtray outside a train station may trigger intrusive thoughts about sensory aspects of smoking and lead a smoker to wonder whether there is time for a cigarette.

According to the EI theory, active cognitive engagement, such as through performance of a working memory task, can serve to counteract the ability to elaborate on cue-related stimuli that may lead to relapse. In support of this view, May, Andrade, Panabokke, and Kavanagh (2010) found that smokers who were instructed to engage in coping strategies that included active cognition (vs. "mind wandering") reported fewer smoking-related thoughts and a reduction in craving following urge induction procedures. Thus, when directed to do so, smokers can utilize cognitive strategies to actively diminish the disruptive effects of cigarette-related cues. The demands of the high-load condition may have engendered a similar effect in the present study. That is, rather than making smokers more susceptible to cue-related distraction, the effort to maintain a relatively large amount of information in working memory may have reduced the cognitive resources available to process the task-irrelevant smoking images. Presumably, any such decrease in the selective attention toand mental elaboration of - smoking cues would have been beneficial for performance, broadly consistent with the predictions of EI theory.

Interestingly, this apparent buffering effect only occurred when high task load was paired with smoking cues: Task performance was not facilitated by the presentation of neutral cues during high-load trials. Somewhat paradoxically, smoking cues may have had a unique effect on accuracy under high load because they posed such a salient threat to performance. It is likely that the degree to which smokers can (implicitly or explicitly) alter the way that a challenging task is approached or executed in the face of smoking cues is moderated by several variables, including the availability of cognitive resources (e.g., as determined by individual differences in working memory capacity), the degree of motivation to perform successfully, and attitudes/intentions regarding cigarette use (e.g., whether or not one is trying to quit smoking). For example, increases in load can only buttress performance against distraction by smoking cues up to a certain point, beyond which the effect plateaus or attenuates as capacity limits are exceeded (Klingberg, 2000). In the context of working memory tasks, the timing of drug-related distractors also appears to be important (e.g., whether they occur during a delay period, as in this study, or during retriev$\mathrm{al}$, as in the study on cue-related distraction in cocaine users conducted by Hester \& Garavan, 2009). Studies that identified and characterized the various parameters that moderate the effects of drug cues on cognitive performance would be valuable.

With respect to neural activation, we found a significant interaction between memory load and delay condition in the rIFG. Delay-related activation of the rIFG did not differ as a function of memory load for control trials or trials containing 
neutral cue distractors. In contrast, activation of the rIFG was significantly greater in the low-memory-load condition relative to the high-memory-load condition for trials containing smoking cue distractors. Furthermore, for trials containing smoking cues, the effect of memory load on behavioral performance (i.e., the buffering effect described above) was partially mediated by the effect of load on delay-related activation of the rIFG. The inferior frontal gyrus (particularly the rIFG) has been implicated in response inhibition and selective attention (Aron, Robbins, \& Poldrack, 2004; Hampshire, Chamberlain, Monti, Duncan, \& Owen, 2010; Prisciandaro et al., 2014). In a study by Hampshire et al. (2010), activation of the rIFG was associated with the detection of important cues, irrespective of whether motor inhibition was required. Therefore, activation of the rIFG is not only associated with inhibiting a response, but is also sensitive to the significance attributed to a stimulus. Of particular relevance to the present study, rIFG activation has also been associated with drug cue reactivity in addicted individuals (Chase, Eickhoff, Laird, \& Hogarth, 2011; Due, Huettel, Hall, \& Rubin, 2002).

Previous research suggested that increases in rIFG activation are an indicator of the degree to which there is a need to prevent attentional capture by irrelevant stimuli when cognitive demands are high. For example, de Fockert and Theeuwes (2012) utilized a combined working memory and visual search task under low and high cognitive loads, with the presence of a singleton distractor during the maintenance period. Although the presence of the distractor did not impact task performance, the rIFG specifically showed greater activation when the singleton was present, but only in the high working memory load condition. The authors concluded that rIFG activation reflects a process of monitoring for and detecting potential distraction (de Fockert \& Theeuwes, 2012). Contrary to the distractor effect observed by de Fockert and Theeuwes, in the present study, rIFG activation was elevated when smoking cues were presented under low, but not under high, memory load. Unlike singleton distractors, the interfering stimuli used in the present experiment were highly salient and personally relevant to participants, which may have contributed to this discrepancy. One possibility is that the elevated rIFG activation observed during smoking cue trials under low load reflects the salience associated with the cigarette-related cues (unlike the neutral images, which were not personally relevant) and/or the need to prevent such stimuli from disrupting performance. In contrast, as would be predicted by EI theory, the cognitive demands of the high-load condition may have reduced the resources available to process the salient distractors, thereby decreasing the need to monitor or inhibit cue-elicited responses. This possibility is reinforced by the observation that load-related changes in activation of the rIFG partially mediated load-related changes in task performance for the trials containing smoking cue distractors.
It is important to note that our neutral and smoking distractors were not associated with differential activation of limbic or reward-related brain regions (e.g., the striatum). Although this may raise questions about the salience of the smoking cues in the present study, the smoking images used as distractors were drawn from a validated set of images that have proven effective for inducing elevated craving and limbic activation in daily smokers in several smoking cue exposure studies (e.g., David et al., 2005; McClernon et al., 2009). Most of this research differed from the present study, however, in that the smoking cues typically were not presented in the context of a cognitive task. Rather, participants were asked to passively view the images or to make simple judgments about them that did not require the active maintenance of information (e.g., identifying the gender of individuals in the images). One possibility is that performing the working memory task (even in the low-load condition) was sufficient to dampen or prevent the activation of reward-related brain regions in response to cigarette-related cues; inclusion of a less demanding task may have allowed for the detection of reward-related activation in response to smoking stimuli. Nonetheless, the pattern of behavioral and neural results clearly suggests that trials containing smoking images were processed differently from both those containing neutral images and those devoid of distractors (control trials).

Additional limitations of the present study should be mentioned. Most importantly, the findings reported above assumed that participants were actively engaged in the task and attending to the distractor stimuli. Although it is possible that participants were using strategies (e.g., closing eyes) to avoid processing the smoking distractors, the participants were instructed they would be asked questions about the pictures, and no participants reported falling asleep during the scan. In addition, both the neutral and smoking-cue distractor conditions - but not the control condition - were associated with robust activation of visual cortical regions, suggesting that participants had viewed the distracting images as instructed. The distractor smoking images were drawn from a standardized set of smoking cues and were matched with comparable neutral images; however, individual craving ratings for the individual smoking cues were not recorded. The present study was not designed to measure subtle changes in craving immediately following exposure to smoking cues during the working memory task. Accordingly, we were not able to explore the possibility that the observed interaction between memory load and distractor condition was related to transient changes in craving (e.g., as a result of decreased attention to the smoking-related stimuli in the high-memory-load condition). The behavioral and neural effects of primary interest were not associated with the urge ratings collected just prior to or after scanning. These constraints could be addressed in future research that incorporated a finer-grained assessment of urge intensity. Relatedly, the CO cutoff employed with the 
present sample may not have been sensitive enough to ensure $12 \mathrm{~h}$ of abstinence. Also, the distractor images were presented only during the maintenance phase of the task. To better understand the relationship between smoking cues and cognitive resources, exposing participants to distractor images during other phases of working memory (e.g., during encoding) would be informative. The sample was also explicitly not treatment-seeking; therefore, caution is warranted in generalizing these results to smokers during a quit attempt. Finally, the present sample was relatively small, and a larger sample will be required to adequately assess for possible age and sex differences, as well as to explore the relationships between couse of cigarettes and other drugs of abuse (e.g., alcohol and cannabis) that were not assessed in the present study.

In summary, the relationship between working memory load and selective attention to salient cues is undoubtedly complex and involves multiple cognitive systems. Much of the existing research has highlighted greater distractibility under high cognitive load, but the alternative (i.e., less susceptibility to distraction under high cognitive load) has also been reported. In the present study, the presentation of taskirrelevant smoking cues during the active maintenance of verbal information reduced the impact of increasing memory load on behavioral performance. This effect was partially mediated by load-related changes in activation of the rIFG. Although these results are surprising, they add to emerging evidence supporting the use of cognitive engagement, via working memory, as a potential strategy for mitigating the impact of smoking cues. Efforts to extend these findings will provide important information for devising strategies to reduce the distracting influence of smoking cues when smokers are confronted with high task demands, such as during a quit attempt.

Author note R.R.M. is currently supported by the Department of Veterans Affairs Office of Academic Affiliations Advanced Fellowship Program in Mental Illness Research and Treatment; the Department of Veterans Affairs New England Mental Illness Research, Education, and Clinical Center (MIRECC); and the VA Connecticut Healthcare System. S.J.W.'s research is supported by the National Institute on Drug Abuse (R21DA037149, R03DA035929) and the National Cancer Institute (R21CA190093). The content is solely the responsibility of the authors and does not necessarily represent the official views of the Department of Veterans Affairs or the National Institutes of Health. We thank the students and staff of the Penn State Addiction, Smoking, and Health Laboratory for their assistance with data collection.

\section{References}

Aron, A. R., Robbins, T. W., \& Poldrack, R. A. (2004). Inhibition and the right inferior frontal cortex. Trends in Cognitive Sciences, 8, 170 177. doi:10.1016/j.tics.2004.02.010

Benjamini, Y., \& Hochberg, Y. (1995). Controlling the false discovery rate: A practical and powerful approach to multiple testing. Journal of the Royal Statistical Society: Series B, 57, 289-300.
Carter, B. L., \& Tiffany, S. T. (1999). Cue-reactivity and the future of addiction research. Addiction, 94, 349-351.

Cepeda-Benito, A., \& Tiffany, S. T. (1996). The use of a dual-task procedure for the assessment of cognitive effort associated with cigarette craving. Psychopharmacology, 127, 155-163.

Chase, H. W., Eickhoff, S. B., Laird, A. R., \& Hogarth, L. (2011). The neural basis of drug stimulus processing and craving: An activation likelihood estimation meta-analysis. Biological Psychiatry, 70, 785793. doi:10.1016/j.biopsych.2011.05.025

Chein, J. M., \& Fiez, J. A. (2010). Evaluating models of working memory through the effects of concurrent irrelevant information. Journal of Experimental Psychology: General, 139, 117-137. doi:10.1037/ a0018200

Corbetta, M., \& Shulman, G. L. (2002). Control of goal-directed and stimulus-driven attention in the brain. Nature Reviews Neuroscience, 3, 201-215. doi:10.1038/nrn755

Cowan, N. (2005). Working memory capacity. Hove, UK: Psychology Press.

Cox, W. M., Fadardi, J. S., \& Pothos, E. M. (2006). The addiction-Stroop test: Theoretical considerations and procedural recommendations. Psychological Bulletin, 132, 443-476. doi:10.1037/0033-2909. 132.3.443

Cropsey, K. L., Trent, L. R., Clark, C. B., Stevens, E. N., Lahti, A. C., \& Hendricks, P. S. (2014). How low should you go? Determining the optimal cutoff for exhaled carbon monoxide to confirm smoking abstinence when using cotinine as a reference. Nicotine \& Tobacco Research, 16, 1348-1355. doi:10.1093/ntr/ntu085

David, S. P., Munafò, M. R., Johansen-Berg, H., Smith, S. M., Rogers, R. D., Matthews, P. M., \& Walton, R. T. (2005). Ventral striatum/ nucleus accumbens activation to smoking-related pictorial cues in smokers and nonsmokers: A functional magnetic resonance imaging study. Biological Psychiatry, 58, 488-494. doi:10.1016/j.biopsych. 2005.04.028

Day, A. M., Kahler, C. W., Metrik, J., Spillane, N. S., Tidey, J. W., \& Rohsenow, D. J. (2015). Working memory moderates the association between smoking urge and smoking lapse behavior after alcohol administration in a laboratory analogue task. Nicotine \& Tobacco Research, 17, 1173-1177. doi:10.1093/ntr/ntu259

de Fockert, J. W. (2013). Beyond perceptual load and dilution: A review of the role of working memory in selective attention. Frontiers in Psychology, 4, 287. doi:10.3389/fpsyg.2013.00287

de Fockert, J. W., Rees, G., Frith, C., \& Lavie, N. (2004). Neural correlates of attentional capture in visual search. Journal of Cognitive Neuroscience, 16, 751-759. doi:10.1162/089892904970762

de Fockert, J. W., \& Theeuwes, J. (2012). Role of frontal cortex in attentional capture by singleton distractors. Brain and Cognition, 80, 367-373. doi:10.1016/j.bandc.2012.07.006

Due, D. L., Huettel, S. A., Hall, W. G., \& Rubin, D. C. (2002). Activation in mesolimbic and visuospatial neural circuits elicited by smoking cues: Evidence from functional magnetic resonance imaging. American Journal of Psychiatry, 159, 954-960.

Ehrman, R. N., Robbins, S. J., Bromwell, M. A., Lankford, M. E., Monterosso, J. R., \& O'Brien, C. P. (2002). Comparing attentional bias to smoking cues in current smokers, former smokers, and nonsmokers using a dot-probe task. Drug and Alcohol Dependence, 67, 185-191.

Engelmann, J. M., Versace, F., Robinson, J. D., Minnix, J. A., Lam, C. Y., Cui, Y., . . . Cinciripini, P. M. (2012). Neural substrates of smoking cue reactivity: A meta-analysis of fMRI studies. NeuroImage, 60, 252-262. doi:10.1016/j.neuroimage.2011.12.024

Evans, D. E., Craig, C., Oliver, J. A., \& Drobes, D. J. (2011). The smoking $N$-back: A measure of biased cue processing at varying levels of cognitive load. Nicotine \& Tobacco Research, 13, 88-93. doi:10.1093/ntr/ntq214 
Ferguson, S. G., \& Shiffman, S. (2009). The relevance and treatment of cue-induced cravings in tobacco dependence. Journal of Substance Abuse Treatment, 36, 235-243. doi:10.1016/j.jsat.2008.06.005

Gilbert, D. G., \& Rabinovich, N. E. (1999). Interational smoking image series (with neutral conterparts), version 1.2. Carbondale, Integrative Neuroscience Laboratory, Department of Psychology, Southern Illinois University.

Goebel, R., Esposito, F., \& Formisano, E. (2006). Analysis of functional image analysis contest (FIAC) data with brainvoyager QX: From single-subject to cortically aligned group general linear model analysis and self-organizing group independent component analysis. Human Brain Mapping, 27, 392-401. doi:10.1002/hbm.20249

Hampshire, A., Chamberlain, S. R., Monti, M. M., Duncan, J., \& Owen, A. M. (2010). The role of the right inferior frontal gyrus: Inhibition and attentional control. NeuroImage, 50, 1313-1319. doi:10.1016/j. neuroimage.2009.12.109

Hawkshead, B., Liebel, S., Schwarz, N., \& Sweet, L. (2014). Working memory and fMRI correlates of smoking cessation treatment outcome. Archives of Clinical Neuropsychology, 29, 577. doi:10.1093/ arclin/acu038.193

Heatherton, T. F., \& Wagner, D. D. (2011). Cognitive neuroscience of self-regulation failure. Trends in Cognitive Sciences, 15, 132-139. doi:10.1016/j.tics.2010.12.005

Heishman, S. J., Boas, Z. P., Hager, M. C., Taylor, R. C., Singleton, E. G., $\&$ Moolchan, E. T. (2006). Effect of tobacco craving cues on memory encoding and retrieval in smokers. Addictive Behaviors, 31, 1116-1121. doi:10.1016/j.addbeh.2005.08.007

Hester, R., \& Garavan, H. (2009). Neural mechanisms underlying drugrelated cue distraction in active cocaine users. Pharmacology Biochemistry and Behavior, 93, 270-277. doi:10.1016/j.pbb.2008. 12.009

Hofmann, W., Friese, M., Schmeichel, B. J., \& Baddeley, A. D. (2011). Working memory and self-regularion. In K. D. Vohs \& R. F. Baumeister (Eds.), Handbook of self regulation: Research, theory, and applications (Vol. 2, pp. 204-225). New York, NY: Guilford Press.

Hopfinger, J. B., Woldorff, M. G., Fletcher, E. M., \& Mangun, G. R. (2001). Dissociating top-down attentional control from selective perception and action. Neuropsychologia, 39, 1277-1291. doi:10. 1016/S0028-3932(01)00117-8

Ilkowska, M., \& Engle, R. W. (2010). Working memory capacity and self-regulation. In R. H. Hoyle (Ed.), Handbook of personality and self-regulation (pp. 265-290). Oxford, UK: Wiley-Blackwell.

Judd, C. M., Kenny, D. A., \& McClelland, G. H. (2001). Estimating and testing mediation and moderation in within-subject designs. Psychological Methods, 6, 115-134. doi:10.1037/1082-989X. 6.2 .115

Kavanagh, D. J., Andrade, J., \& May, J. (2005). Imaginary relish and exquisite torture: The elaborated intrusion theory of desire. Psychological Review, 112, 446-467. doi:10.1037/0033-295X.112. 2.446

Klingberg, T. (2000). Limitations in information processing in the human brain: Neuroimaging of dual-task performance and working memory tasks. Progress in Brain Research, 126, 95-102.

Lavie, N., Hirst, A., de Fockert, J. W., \& Viding, E. (2004). Load theory of selective attention and cognitive control. Journal of Experimental Psychology: General, 133, 339-354. doi:10.1037/0096-3445. 133.3.339

Loughead, J., Wileyto, E. P., Ruparel, K., Falcone, M., Hopson, R., Gur, R., \& Lerman, C. (2015). Working memory-related neural activity predicts future smoking relapse. Neuropsychopharmacology, 40, 1311-1320. doi:10.1038/npp.2014.318

Madden, C. J., \& Zwaan, R. A. (2001). The impact of smoking urges on working memory performance. Experimental and Clinical Psychopharmacology, 9, 418-424.
May, J., Andrade, J., Panabokke, N., \& Kavanagh, D. (2010). Visuospatial tasks suppress craving for cigarettes. Behaviour Research and Therapy, 48, 476-485. doi:10.1016/j.brat.2010. 02.001

McClernon, F. J., Kozink, R. V., Lutz, A. M., \& Rose, J. E. (2009). 24-h smoking abstinence potentiates fMRI-BOLD activation to smoking cues in cerebral cortex and dorsal striatum. Psychopharmacology, 204, 25-35. doi:10.1007/s00213-008-1436-9

Nestor, L., McCabe, E., Jones, J., Clancy, L., \& Garavan, H. (2011). Differences in "bottom-up" and "top-down" neural activity in current and former cigarette smokers: Evidence for neural substrates which may promote nicotine abstinence through increased cognitive control. NeuroImage, 56, 2258-2275. doi:10.1016/j.neuroimage. 2011.03.054

Nichols, T. T., \& Wilson, S. J. (2015). Working memory functioning and addictive behavior: Insights from cognitive neuroscience. In S. J. Wilson (Ed.), Wiley handbook on the cognitive neuroscience of addiction (pp. 55-75). Chichester, UK: Wiley.

Patterson, F., Jepson, C., Loughead, J., Perkins, K., Strasser, A. A., Siegel, S., . . . Lerman, C. (2010). Working memory deficits predict short-term smoking resumption following brief abstinence. Drug and Alcohol Dependence, 106, 61-64. doi:10.1016/j.drugalcdep. 2009.07.020

Payne, B. K., McClernon, F. J., \& Dobbins, I. G. (2007). Automatic affective responses to smoking cues. Experimental and Clinical Psychopharmacology, 15, 400-409. doi:10.1037/ 1064-1297.15.4.400

Piasecki, T. M. (2006). Relapse to smoking. Clinical Psychology Review, 26, 196-215. doi:10.1016/j.cpr.2005.11.007

Prisciandaro, J. J., Joseph, J. E., Myrick, H., McRae-Clark, A. L., Henderson, S., Pfeifer, J., \& Brady, K. T. (2014). The relationship between years of cocaine use and brain activation to cocaine and response inhibition cues. Addiction, 109, 2062-2070. doi:10.1111/ add. 12666

Sandberg, A., Sköld, C. M., Grunewald, J., Eklund, A., \& Wheelock, Å. M. (2011). Assessing recent smoking status by measuring exhaled carbon monoxide levels. PLoS ONE, 6(28864), 1-7. doi:10.1371/ journal.pone. 0028864

Sayette, M. A., \& Hufford, M. R. (1994). Effects of cue exposure and deprivation on cognitive resources in smokers. Journal of Abnormal Psychology, 103, 812-818. doi:10.1037/0021-843X.103.4.812

Sayette, M. A., Martin, C. S., Wertz, J. M., Shiffman, S., \& Perrott, M. A. (2001). A multi-dimensional analysis of cue-elicited craving in heavy smokers and tobacco chippers. Addiction, 96, 1419-1432. doi:10.1080/09652140120075152

Sayette, M. A., \& Tiffany, S. T. (2013). Peak provoked craving: An alternative to smoking cue-reactivity. Addiction, 108, 1019-1025. doi:10.1111/j.1360-0443.2012.04013.x

Shiffman, S., Paty, J. A., Gnys, M., Kassel, J. A., \& Hickcox, M. (1996). First lapses to smoking: Within-subjects analysis of real-time reports. Journal of Consulting and Clinical Psychology, 64, 366-379.

Talairach, J., \& Tournoux, P. (1988). Co-planar stereotaxic atlas of the human brain: An approach to medical cerebral imaging. Stuttgart, Germany: Thieme.

Tiffany, S. T., \& Drobes, D. J. (1990). Imagery and smoking urges: The manipulation of affective content. Addictive Behaviors, 15, 531-539.

Wagner, D. D., Dal Cin, S., Sargent, J. D., Kelley, W. M., \& Heatherton, T. F. (2011). Spontaneous action representation in smokers when watching movie characters smoke. Journal of Neuroscience, 31, 894-898. doi:10.1523/JNEUROSCI.5174-10.2011

Waters, A. J., Shiffman, S., Sayette, M. A., Paty, J. A., Gwaltney, C. J., \& Balabanis, M. H. (2004). Cue-provoked craving and nicotine replacement therapy in smoking cessation. Journal of Consulting and Clinical Psychology, 72, 1136-1143. doi:10.1037/0022-006X. 72.6.1136 
Wilson, S. J., Sayette, M. A., \& Fiez, J. A. (2012). Quitting-unmotivated and quitting-motivated cigarette smokers exhibit different patterns of cue-elicited brain activation when anticipating an opportunity to smoke. Journal of Abnormal Psychology, 121, 198-211. doi:10. 1037/a0025112

Wilson, S. J., Sayette, M. A., Fiez, J. A., \& Brough, E. (2007). Carry-over effects of smoking cue exposure on working memory performance. Nicotine \& Tobacco Research, 9, 613-619. doi:10.1080/ 14622200701243144
Yoon, J. H., Curtis, C. E., \& D’Esposito, M. (2006). Differential effects of distraction during working memory on delay-period activity in the prefrontal cortex and the visual association cortex. NeuroImage, 29, 1117-1126. doi:10.1016/j.neuroimage.2005.08.024

Zarahn, E., Aguirre, G. K., \& D'Esposito, M. (1999). Temporal isolation of the neural correlates of spatial mnemonic processing with fMRI. Cognitive Brain Research, 7, 255-268. doi:10.1016/S09266410(98)00029-9 